\title{
Management of Intrapleural Sepsis with Once Daily Use of Tissue Plasminogen Activator and Deoxyribonuclease
}

\author{
Hiren J. Mehta ${ }^{a}$ Abhishek Biswas ${ }^{a}$ Andrea M. Penley ${ }^{a}$ Jessica Cope ${ }^{b}$ \\ Matthew Barnes ${ }^{\mathrm{a}}$ Michael A. Jantz ${ }^{\mathrm{a}}$ \\ aDivision of Pulmonary, Critical Care, and Sleep Medicine, University of Florida College of Medicine, and \\ ${ }^{\mathrm{b}}$ Department of Pharmacy, University of Florida Health Shands Hospital, Gainesville, Fla., USA
}

For editorial comment see p. 99

\section{Key Words}

Tissue plasminogen activator - Deoxyribonuclease .

Empyema $\cdot$ Complicated parapneumonic effusion

\begin{abstract}
Background: Pleural infection remains a significant cause of morbidity, mortality, prolonged hospital stay, and increased healthcare costs, despite advances in therapy. Twice daily intrapleural tissue plasminogen activator (tPA)/deoxyribonuclease (DNase) initiated at the time of diagnosis has been shown to significantly improve radiological outcomes and decrease the need for surgery. Objectives: To analyze our experience with once daily tPA/DNase for intrapleural sepsis. Methods: Data derived from consecutive patients with empyema and complicated parapneumonic effusion who received once daily intrapleural tPA/DNase between January 2012 and August 2014 were reviewed. Measured outcomes included treatment success at 30 days, volume of pleural fluid drained, improvement in radiographic pleural opacity, length of hospital stay, need for surgery, and adverse events. Results: 55 consecutive patients ( 33 male; mean age \pm SD, $54.6 \pm 16.1$ years) were treated with once daily intrapleural tPA/DNase for 3 days. The majority of the patients $(n=51$; 92.7\%) were successfully managed without the need for sur-
\end{abstract}

\section{KARGER}

E-Mail karger@karger.com

www.karger.com/res gical intervention. The mean change in pleural opacity measured on chest radiograph at day 7 was $-28.8 \pm 17.6 \%$. The median amount of fluid drained was $2,195 \mathrm{ml}$. No serious adverse events requiring discontinuation of intrapleural medications were observed. The most common complication was pain requiring escalating doses of analgesics $(n=8$; 15\%). Compliance with the protocol was excellent. Conclusion: Early administration of once daily intrapleural tPA/ DNase for 3 days is safe, effective, and represents a viable option for the management of empyema and complicated parapneumonic effusion.

(c) 2016 S. Karger AG, Basel

\section{Introduction}

Pleural infection remains a common and serious respiratory condition with important implications for patients. Over 80,000 new cases of pleural infection are diagnosed in adults in the UK and US each year with a cost of over USD 500 million $[1,2]$. Infected pleural fluid is loculated by septations and may be highly viscous, rendering drainage difficult. Surgical drainage is often necessary. Although some observational studies have shown that intrapleural administration of fibrinolytic drugs re- 
duced the frequency of failed drainage and the need for subsequent surgery [3-8], the large Multicenter Intrapleural Sepsis Trial (MIST)-1 [9] showed no benefit from intrapleural streptokinase. A subsequent meta-analysis, which included MIST-1, revealed that intrapleural fibrinolytic therapy was associated with a nonsignificant reduction in death and the need for surgery when compared with placebo [10].

In search of methods to improve pleural fluid drainage, efforts have been focused on targeting pleural fluid viscosity, believed to arise from DNA liberated by degranulated leukocytes. Recombinant deoxyribonuclease (DNase), which effectively fragments free uncoiled DNA in pus, has been used for decades to enhance sputum clearance in patients with cystic fibrosis. DNase, when applied to pleural pus ex vivo, potently enhanced its drainage [11]. The combined use of fibrinolytics and DNase has been shown to have synergistic action in an animal model of empyema [12]. A recent multicenter randomized controlled trial showed that combination intrapleural therapy with tissue plasminogen activator (tPA) and DNase improved drainage of infected effusions, and improved secondary outcomes including the need for surgical intervention and the length of hospital stay (MIST-2) [13].

Nevertheless, several uncertainties about intrapleural tPA/DNase persist. The appropriate intrapleural dose of tPA/DNase for individual patients is not known, nor has dosing frequency been compared. The intrapleural tPA/ DNase dosing regimen used in MIST-2 was empirically based on early case reports $[14,15]$. Once daily administration of urokinase has been shown to have similar efficacy as twice daily dosing for empyema in children [16]. It is unknown whether this data can be extrapolated to once versus twice daily dosing of tPA/DNase for empyema or complicated parapneumonic effusions in adults.

At our institution, we have used a 3-day regimen of once daily tPA/DNase to treat intrapleural sepsis. The aim of the current work is therefore to report our experience with once daily tPA/DNase for 3 days, initiated within $24 \mathrm{~h}$ of chest drain placement, in patients with empyema and complicated parapneumonic effusion.

\section{Methods}

Consecutive patients receiving intrapleural tPA/DNase for pleural space infection between January 2012 and August 2014 were prospectively recorded and relevant data were extracted retrospectively. All research and analysis was approved by the University of Florida's Institutional Review Board (No. 201400654).
Pleural space infection was defined, consistent with previous studies [9], to include both complicated parapneumonic effusion (fluid $\mathrm{pH} \leq 7.2$ in a patient with clinical evidence of infection) and empyema (macroscopic pus and/or the presence of bacteria on pleural fluid Gram's stain or culture). Exclusion criteria included: age $<18$ years; previous treatment with intrapleural fibrinolytic agents, DNase, or both for empyema; known sensitivity to DNase or tPA; coincidental stroke; major hemorrhage or major trauma; major surgery in the previous 5 days; previous pneumonectomy on the infected side; pregnancy or lactation; and expected survival $<3$ months, owing to a condition other than that responsible for the pleural space infection.

The first doses were administered within $24 \mathrm{~h}$ of pleural drain placement. Both $10 \mathrm{mg}$ tPA and $5 \mathrm{mg}$ DNase (each in $30 \mathrm{ml}$ of $0.9 \%$ $\mathrm{NaCl}$ ) were instilled intrapleurally once daily for a total of 3 doses over 3 consecutive days. The protocol was as follows: first, tPA was instilled via the chest tube, followed by a $10-25 \mathrm{ml}$ flush of $0.9 \%$ $\mathrm{NaCl}$. The chest tube was then clamped for $60 \mathrm{~min}$ before unclamping and connecting it to wall suction at $-20 \mathrm{~cm} \mathrm{H}_{2} \mathrm{O}$, allowing free drainage for $60 \mathrm{~min}$. The procedure was then repeated with DNase. To promote adequate drainage, negative pressure $\left(-20 \mathrm{~cm} \mathrm{H}_{2} \mathrm{O}\right)$ was consistently applied to the pleural drain when it was unclamped. Daily chest radiographs (CXRs) were obtained for the first 7 days. Inpatient management, including anti-infective selection and duration of therapy, was at the discretion of the attending physician.

The patient records were interrogated for patient demographics, treatment details (e.g., timing of therapy), and outcomes including volume of fluid drained, adverse events, length of hospital stay, need for surgery, and mortality. Laboratory data, specifically pleural fluid biochemistry and microbiologic cultures, were recorded. The area of pleural opacity, expressed as the percentage of the ipsilateral hemithorax occupied by effusion, was quantified on CXR at day 1 and day 7 by one investigator using Image J software [17] as previously described, and was precisely replicated as such [13]. The area of pleural opacity and the area of the ipsilateral hemithorax were measured digitally. Change in pleural opacity was measured by subtracting the percentage of ipsilateral hemithorax occupied by pleural opacity at day 7 from the percentage occupied at day 1 .

Treatment success was defined as survival to discharge and avoidance of need for surgical intervention for pleural infection within the first 30 days after the treatment. We also analyzed the change in the area of pleural opacity, the volume of pleural fluid drained, the length of stay from first dose of tPA/DNase to hospital discharge or death, and the frequency of serious and non-serious adverse events, including major bleeding requiring blood transfusion, or any other event requiring cessation of intrapleural therapy during the study period, and report those here. All variables were analyzed using simple descriptive statistics.

\section{Results}

A total of 55 consecutive patients (33 male; mean age $\pm \mathrm{SD}, 55 \pm 16$ years) with empyema or complicated parapneumonic effusions were analyzed. The majority of the patients had significant medical comorbidities $(46 / 55)$ as described in table 1 . Forty of the 55 patients $(73 \%)$ had 
Table 1. Patient comorbidities

\begin{tabular}{lr}
\hline Comorbidity & $\mathrm{n}$ \\
\hline Hypertension & 6 \\
HIV & 4 \\
Connective tissue diseases & 2 \\
Asthma & 2 \\
Diabetes Mellitus & 12 \\
Coronary artery disease & 2 \\
Intravenous drug abuse & 2 \\
Cystic fibrosis & 1 \\
Chronic kidney disease & 3 \\
Cirrhosis & 3 \\
Hepatitis C & 1 \\
Stroke & 5 \\
Chronic obstructive lung disease & 8 \\
Malignancy & 10 \\
\hline
\end{tabular}

empyema; the remainders were diagnosed with complicated parapneumonic effusion. Thirty-five patients (64\%) presented with right-sided effusion, and the remaining 20 patients (36\%) were affected on the left side. Thirty-four patients had microbiologically positive pleural fluid cultures (table 2).

Forty-six patients (85\%) had image-guided pigtail catheter (12 or $14 \mathrm{Fr}$ ) placement to drain the infected pleural space, whereas the remaining 9 patients $(15 \%)$ had surgical chest tubes. The median total duration of chest tube drainage was 9 days [interquartile range (IQR), $7-11]$, and the duration of antibiotic therapy was 28 days (IQR, 18.5-35). The median amount of fluid drained for the duration of chest tube placement was 2,195 ml (IQR, 1,473-3,505). Table 3 contains patient demographics, pleural fluid characteristics, and treatment details.

\section{Treatment Success}

Fifty-one of 55 patients $(92.7 \%)$ were successfully treated with intrapleural tPA/DNase. Four patients $(7.3 \%)$ had disease progression requiring surgery despite intrapleural therapy. Three patients died within 30 days after intrapleural therapy. In all three cases, sepsis due to pleural infection had been controlled; however, the patients died from their underlying comorbidities. One patient died following surgery and the other two due to underlying comorbidity (malignancy). In both of those cases, sepsis was controlled but prolonged hospital stay and generalized deconditioning led to the election of palliative care measures. Using the composite endpoint of survival and discharge from hospital without necessitating surgical inter-
Table 2. Microbiology of the pleural fluid

\begin{tabular}{ll}
\hline Microorganism cultured in pleural fluid & $\mathrm{n}$ \\
\hline Hemophilus influenza & 1 \\
Corynebacterium & 1 \\
Proteus mirabilis & 2 \\
Mixed anaerobe & 2 \\
Beta-hemolytic streptococci group C & 2 \\
Methicillin-sensitive Staphylococcus aureus & 3 \\
Streptococci pneumonia & 4 \\
Microaerophilic streptococci & 6 \\
Methicillin-resistant Staphylococcus aureus & 2 \\
Escherichia coli & 2 \\
Pseudomonas aeruginosa & 3 \\
Mixed infections with more than one organism & 5 \\
Mycobacterium fortuitum & 1 \\
\hline
\end{tabular}

Table 3. Demographic, pleural fluid, and treatment characteristics

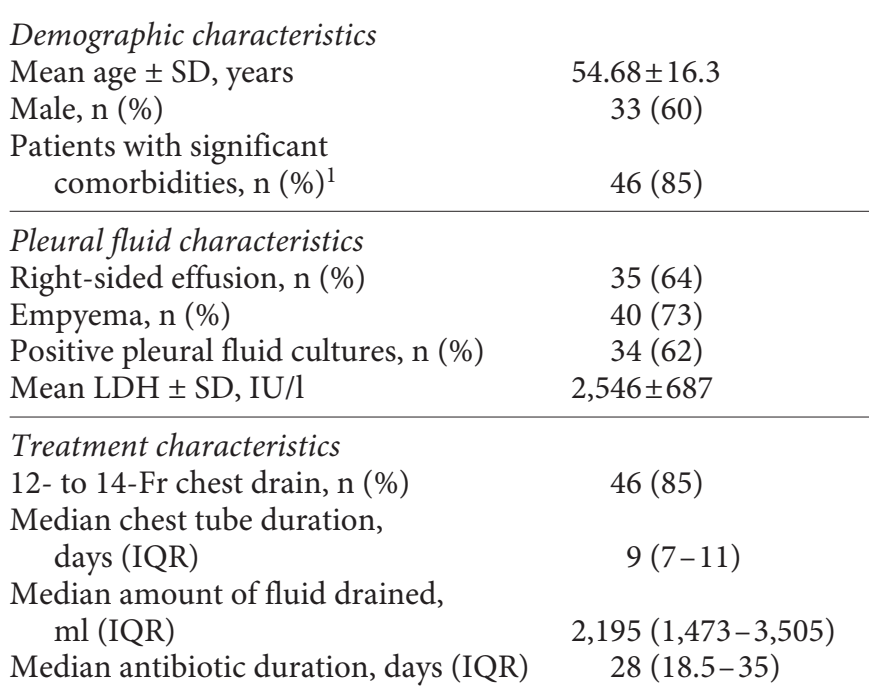

${ }^{1}$ For details, see table 1.

vention at 30 days, 48 patients $(87.3 \%)$ had complete success from intrapleural tPA/DNase treatment.

Indications for surgery included persistent fluid collection observed on imaging and/or failure to respond to treatment with persistent sepsis $(n=2)$, and inability of the lung to fully re-expand, leaving a potential space for recurrent infection $(\mathrm{n}=2)$. One of the four patients who underwent surgery died within 30 days of intrapleural tPA/DNase instillation. 


\section{Radiographic Changes}

The degree of pleural opacity on CXR decreased from a mean of $51.5 \pm 19.1 \%$ on day 1 to a mean of $22.6 \pm 15.3 \%$ on day 7 . The mean change in pleural opacity, calculated as the difference between the percentage of hemithorax occupied on day 1 and day 7 , was $-28.8 \pm 17.6 \%$. Figure 1 shows the percentage volume of hemithorax occupied by pleural effusion on day 1 and day 7 amongst all patients. The decrease in volume at day 7 was statistically significant $(\mathrm{p}<0.0001)$.

\section{Fluid Drainage}

Most patients were managed with small bore chest tubes, 12-14 Fr in size. The median chest tube duration was 9 days (IQR, 7-11), and the median amount of fluid drained for the chest tube duration was $2,195 \mathrm{ml}$ (IQR, $1,473-3,505)$.

\section{Adverse Events}

There were no major bleeding events requiring blood transfusion or discontinuation of therapy. Eight patients (15\%) had worsening chest pain along the pleural drain site requiring increasing doses of pain medications within $24 \mathrm{~h}$ of intrapleural therapy. Two patients (4\%) had erythema and swelling along the drainage site. No other serious or non-serious adverse events were noted.

\section{Duration of Hospital Stay}

The median duration of hospital stay from first dose of tPA/DNase to hospital discharge or death was 13 days (IQR, 11-18).

\section{Discussion}

Our study shows that once daily intrapleural tPA/ DNase for 3 consecutive days in patients with empyema and complicated parapneumonic effusion appears to be safe and effective. In our cohort of patients, treatment proved to be successful by avoidance of surgery in $92.7 \%$ of the patients. The primary endpoint, survival at 30 days without necessitating surgery, was achieved in $87.3 \%$ of the patients. Furthermore, although external comparisons cannot be made, the results from our study with regards to survival, rates of surgery, amount of fluid drained, radiographic changes, and length of hospital stay appeared similar to those mentioned in previous studies using twice daily intrapleural tPA/DNase $[13,18]$. The treatment was well tolerated, with $<20 \%$ of the patients requiring escalation of baseline analgesia. There were no

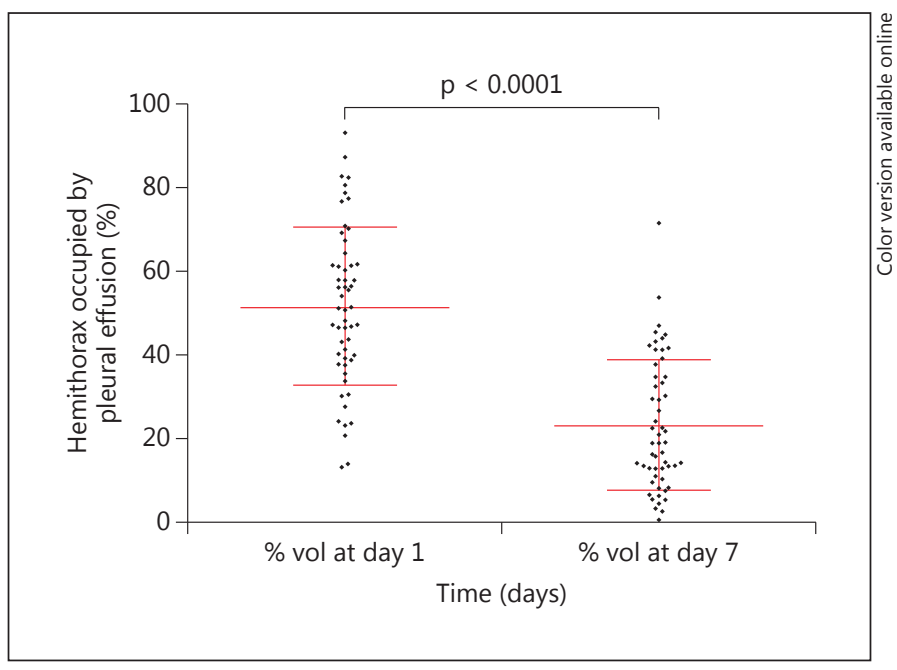

Fig. 1. Percentage volume of hemithorax occupied by pleural effusion on day 1 and day 7 . The decrease in volume at day 7 was statistically significant $(\mathrm{p}<0.0001)$.

major adverse events, and the compliance with a once daily regimen for 3 days was excellent with none of the patients requiring discontinuation of therapy.

Despite advances in care, mortality from pleural infection remains high at approximately 15\% [9]. Standard treatment for pleural infection includes prompt drainage and appropriate antibiotic therapy $[19,20]$, which is empirical in up to $40 \%$ of all cases, as the causative organisms remain microbiologically obscure [21]. Medical management fails in around $25 \%$ of all cases and requires surgical drainage [9]. A recent study by Rahman et al. [13] showed that the sequential use of intrapleural tPA/DNase twice daily for 3 days in these patients resulted in a significant reduction in surgical referral and length of hospital stay, leading to a paradigm shift in the way these patients are managed. A subsequent study by Piccolo et al. [18] confirmed that the delayed administration of twice daily intrapleural tPA/DNase had similar improved outcomes.

Our rationale for administering a once daily regimen was several fold. A consensus regarding the dose and frequency of intrapleural fibrinolytic therapy is lacking [22]. Reported intrapleural fibrinolytic dosing frequency has ranged from once to twice daily $[23,24]$. Administration frequency appears to be independent of the agent's short half-life of elimination and rather targeted at breaking up intrapleural septations. In 2006, Wang et al. [16] evaluated once versus twice daily administration of urokinase. No significant differences were observed with regards to length of hospital stay, amount of pleural fluid drained, 
need for surgical intervention, or treatment failure [16]. Outcomes for a once daily regimen with $\mathrm{tPA} / \mathrm{DNase}$ were fairly similar to the twice daily regimen in our study.

Twice daily tPA/DNase involves a significant time commitment from the providers, especially at institutions where bedside nurses are not credentialed to administer intrapleural medications. This can lead to decreased compliance with a twice daily regimen, as is evident by a $71 \%$ compliance rate in the MIST-2 trial [13]. In the real world setting, this has the potential to be even lower. Our study had $100 \%$ compliance with the once daily regimen. The potential for serious adverse events may also be mitigated with a once daily regimen. No patients included in our study cohort experienced significant pleural or systemic bleeding requiring discontinuation of therapy or additional intervention. We thus feel that once daily administration of intrapleural tPA/DNase appears to be safe and suggests similar clinically efficacy as twice daily administration.

Once daily administration is economically advantageous. Per our institution-specific cost analysis, once daily intrapleural administration of $10 \mathrm{mg}$ tPA followed by $5 \mathrm{mg}$ DNase would result in a cost savings of USD 2,657.14 per 3-day course when compared to twice daily administration. The cost savings may be even higher considering alteplase's short stability and how the product is supplied (USD 3,552.47/50 mg vial); frequently, the remaining 40 $\mathrm{mg}$ is wasted. Furthermore, this calculation accounts for cost savings from medications alone and does not take into consideration personnel time and effort and other costs that may be involved with twice daily administration of intrapleural medications.

Limitations of our study are that it is a retrospective series of prospectively collected data and direct compari- sons between once and twice daily regimens cannot be made. Due to the retrospective nature of the study, there were no predefined criteria for progression to surgery. All treatment decisions were made by the attending physician, which may have resulted in heterogeneity in management. However, such an approach validates the effectiveness of tPA/DNase in a 'real-life' setting. This case series therefore supports the sequential use of intrapleural tPA/DNase and makes a strong case for early initiation, within the first $24 \mathrm{~h}$, of a once daily regimen for 3 consecutive days. Our study is also the largest series of sequential use of intrapleural tPA/DNase in the US to date.

Future studies are needed to compare the once daily to twice daily regimen in a randomized prospective fashion. Investigation comparing initiation of this regimen within $24 \mathrm{~h}$, versus as a salvage modality for patients who fail standard drainage and antibiotics, is also needed. The optimal role of $\mathrm{tPA} / \mathrm{DNase}$ in the treatment algorithm for pleural infection needs to be determined. Video-assisted thoracoscopic surgery remains the preferred treatment for pleural infection in many centers. Direct comparison of video-assisted thoracoscopic surgery with intrapleural tPA/DNase should be evaluated as well.

In summary, this study shows that sequential administration of once daily intrapleural tPA/DNase for 3 days is safe and effective, it may be a reasonable alternative to a twice daily regimen, and it may be associated with fewer serious adverse events.

\section{Financial Disclosure and Conflicts of Interest}

No potential conflicts of interest exist with any companies/organizations whose products or services are discussed in this article.

\section{References}

1 Grijalva CG, et al: Emergence of parapneumonic empyema in the USA. Thorax 2011;66: 663-668.

2 Sonnappa S, et al: Comparison of urokinase and video-assisted thoracoscopic surgery for treatment of childhood empyema. Am J Respir Crit Care Med 2006;174:221-227.

-3 Davies RJ, Traill ZC, Gleeson FV: Randomised controlled trial of intrapleural streptokinase in community acquired pleural infection. Thorax 1997;52:416-421.

4 Bouros D, et al: Intrapleural urokinase versus normal saline in the treatment of complicated parapneumonic effusions and empyema. A randomized, double-blind study. Am J Respir Crit Care Med 1999;159:37-42. $\checkmark 5$ Tuncozgur B, et al: Intrapleural urokinase in the management of parapneumonic empyema: a randomised controlled trial. Int J Clin Pract 2001;55:658-660.

6 Talib SH, et al: Utility of intrapleural streptokinase in management of chronic empyemas. J Assoc Physicians India 2003;51:464-468.

7 Diacon AH, et al: Intrapleural streptokinase for empyema and complicated parapneumonic effusions. Am J Respir Crit Care Med 2004;170:49-53.

$\checkmark 8$ Misthos P, et al: Early use of intrapleural fibrinolytics in the management of postpneumonic empyema. A prospective study. Eur J Cardiothorac Surg 2005;28:599-603.
9 Maskell NA, et al: UK Controlled trial of intrapleural streptokinase for pleural infection. N Engl J Med 2005;352:865-874.

$\checkmark 10$ Tokuda Y, et al: Intrapleural fibrinolytic agents for empyema and complicated parapneumonic effusions: a meta-analysis. Chest 2006;129:783-790.

11 Simpson G, Roomes D, Heron M: Effects of streptokinase and deoxyribonuclease on viscosity of human surgical and empyema pus. Chest 2000;117:1728-1733.

12 Zhu Z, et al: Tissue plasminogen activator combined with human recombinant deoxyribonuclease is effective therapy for empyema in a rabbit model. Chest 2006;129:1577-1583.
Once Daily tPA/DNase for Pleural Infection
Respiration 2016;91:101-106 DOI: $10.1159 / 000443334$ 
13 Rahman NM, et al: Intrapleural use of tissue plasminogen activator and DNase in pleural infection. N Engl J Med 2011;365:518-526.

14 Simpson G, Roomes D, Reeves B: Successful treatment of empyema thoracis with human recombinant deoxyribonuclease. Thorax 2003;58:365-366.

15 Fujiwara K, et al: Intrapleural streptokinasestreptodornase in the treatment of empyema and hemothorax (in Japanese). Kyobu Geka 2002;55:1115-1119.

16 Wang JN, et al: Once-daily vs. twice-daily intrapleural urokinase treatment of complicated parapneumonic effusion in paediatric patients: a randomised, prospective study. Int J Clin Pract 2006;60:1225-1230.
17 Asband WS: Image J. Bethesda, US National Institutes of Health, 1997-2014. http://imagej.nih.gov/ij/.

18 Piccolo F, et al: Intrapleural tissue plasminogen activator and deoxyribonuclease for pleural infection. An effective and safe alternative to surgery. Ann Am Thorac Soc 2014;11: 1419-1425.

19 Colice GL, et al: Medical and surgical treatment of parapneumonic effusions: an evidence-based guideline. Chest 2000;118:11581171.

20 Davies HE, et al: Management of pleural infection in adults: British Thoracic Society Pleural Disease Guideline 2010. Thorax 2010; 65(suppl 2):ii41-ii53.
21 Maskell NA, et al: The bacteriology of pleural infection by genetic and standard methods and its mortality significance. Am J Respir Crit Care Med 2006; 174:817-823.

22 Janda S, Swiston J: Intrapleural fibrinolytic therapy for treatment of adult parapneumonic effusions and empyemas: a systematic review and meta-analysis. Chest 2012;142:401411.

23 Ahmed AH, Yacoub TE: Intrapleural therapy in management of complicated parapneumonic effusions and empyema. Clin Pharmacol 2010;2:213-212.

24 Froudarakis ME, et al: Recombinant tissue plasminogen activator in the treatment of pleural infections in adults. Respir Med 2008; 102:1694-1700. 